See Article page 224 .

\section{Commentary: Leaders float all boats higher and faster}

\author{
Robert J. Cerfolio, MD, MBA
}

Lo and colleagues ${ }^{1}$ from Duke University have presented an interesting manuscript. I congratulate them on the quality of the writing and statistical analysis and the finished product. The legacy of any study is its main finding and clinical import. The main finding in this study is that opioid use (delivered parenterally) has NOT decreased from 2009 to 2018 on postoperative days $0,1,2$, and 3 after lobectomy. Other findings are that there was an increase in the dosage of parenterally delivered opioids in patients who underwent lobectomy via an open thoracotomy and in video-assisted thoracoscopic platform but not for patients who had robotic surgery. Perhaps the more important question is what is the clinical import of this study?

We suspect that few thoracic surgeons mind if their patient is taking some narcotics on postoperative day $0,1,2$, or 3 after an anatomical pulmonary resection. The key question to pose is as follows: Are there any data that show a link between some narcotics use on postoperative day 0 to 3 and long-term addiction?

The main initiative of limiting narcotic use after surgery is to prevent long-term narcotic addiction. We encourage our patients, the vast majority of whom are now discharged on postoperative day 1 and only receive oral medicines immediately postoperatively, to take a narcotic (opioid) at home, such as oxycodone by mouth, to help sleep each night

\footnotetext{
From the Department of Cardiothoracic Surgery, New York University Langone Health, New York, NY.

Disclosures: Dr Cerfolio discloses no relationships over the last 2.5 years, but past relationships were with AstraZeneca, Bard Davol, Bovie Medical Corporation, CSATS, ConMed, Covidien/Medtronic, Ethicon, Fruit Street Health, Google/Verb Surgical, Intuitive Surgical, KCI/Acelity, Myriad Genetics, Neomend, Pinnacle Biologics, ROLO-7, Tego, and TransEnterix.

The Journal policy requires editors and reviewers to disclose conflicts of interest and to decline handling or reviewing manuscripts for which they may have a conflict of interest. The editors and reviewers of this article have no conflicts of interest.

Received for publication April 7, 2021; revisions received April 19, 2021; accepted for publication April 23, 2021; available ahead of print May 13, 2021.

Address for reprints: Robert J. Cerfolio, MD, MBA, Department of Cardiothoracic Surgery, NYU Langone Health, 530 1st Ave, Suite 9V, New York, NY 10016 (E-mail: robert.cerfolio@nyumc.org).

JTCVS Open 2021;6:237-8

2666-2736

Copyright (c) 2021 The Author(s). Published by Elsevier Inc. on behalf of The American Association for Thoracic Surgery. This is an open access article under the CC BY-NC-ND license (http://creativecommons.org/licenses/by-nc-nd/4.0/).

https://doi.org/10.1016/j.xjon.2021.04.012
}

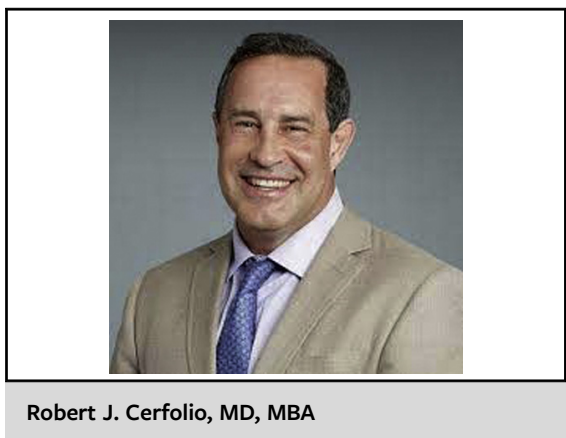

CENTRAL MESSAGE

Let us choose to lead. Let's decide as a group to float all boats higher and faster, so all patients with lung cancer who come to the surgical arena can benefit from optimal outcomes.

for the first several days after surgery. We are not aware of any data that suggest it is addictive. Moreover, in more than 25 years I have not observed chronic addiction from this type of usage that often goes out to postoperative day 5 .

To be scientifically accurate, the aforementioned question we pose may be slightly different from the study's findings, ie, taking opioids by mouth is clearly different from taking it parenterally, but the question remains the same: Is taking "some" opioids on postoperative day $0,1,2$, or 3 a surrogate for addiction? Perhaps as important to ask is the value proposition—does it add value by mitigating less chronic pain? What is the true value of using limited all-opioid use (even one pill a day) when they are supplemented with other nonaddictive analgesic for the first week after surgery?

As the authors adroitly show, most all of us have added many other types of analgesic agents (at least 2 or 3 ) to an opioid in an effort to mitigate addiction. These agents work via different mechanisms to control pain and are not addictive. This may be the best advance over the time frame of this study. The authors have shown this well and described it well in the discussion. In true clinical practice, few of us give more than a 5-day supply (some states make it almost impossible to give more if we wanted to).

The disconcerting quality metrics reported in this paper emphasize that from 2009 to 2018 we-as a collective group of thoracic surgeons-have NOT offered our patients optimal care. To improve outcomes for all patients, this uncomfortable topic should be thoroughly discussed by first 
agreeing on a few principles: (1) a minimally invasive platform is better than open thoracotomy; (2) avoiding an epidural is better; (3) a shorter length of stay is better; and (4) getting patients on medicines by mouth is better.

With regard to points 1 and 2, the authors showed that $41 \%$ of patients underwent open thoracotomy, $42 \%$ had a video-assisted thoracoscopic surgery (VATS), and 17\% had a robotic approach. Our experience at New York University Langone Health System is different inasmuch as, unless the tumor is $10 \mathrm{~cm}$ or greater, a minimally invasive approach with no epidural (unless young patients with preoperative addiction) is routinely recommended. As to points 3 and 4, the authors reported an average length of stay of 6 days for those patients who undergo thoracotomy, 4 days for those who had VATS, and 3 days for those who had robotics. Our target should be striving for a 23-hour length of stay, which is what we are currently aiming for at New York University Langone Health System. Also, the authors' encouraging results on reduced use of epidural-from $15 \%$ to $5 \%$ for VATS and $16 \%$ to $1.3 \%$ for robotics over the time of the study-should serve as the driver for further minimization of its use.

Some may argue that we are being too critical or too tough or setting the bar "too high." We believe if the patient's vantage point is considered we are not. This circles to the main message of this editorial.

We as a group of leaders, as thoracic surgeons, have not taken enough accountability to ensure that all of our patients receive the optimal care that our own systems can deliver. We have opportunity to improve. We know we can do better. We know who in our own system or in nearby systems offers the best care. Yet, we have lacked the collective leadership and transparency and data-sharing. We have chosen rather to avoid the tough conversations to ensure that ALL patients have access to those providers that are more likely to deliver optimal outcomes. And this culture is true in other surgical and medical specialties as well.

Once we decide as a group to leverage honest, accurate, and meaningful metricized data that reflect quality care, patient outcomes will rise high. And all boats will rise quickly. With the reporting of quality measures that compare apples to apples, such as the Efficiency Quality Index, ${ }^{2}$ which leverages verified data with sensible attribution of validated metrics, behavior changes. Patients do better. Doctors do better. Health care does better. The survival from nonsmall lung cancer rises. Once we offer reliable data like those from the Efficiency Quality Index and then present them transparently with one another in an egoless-surgical system that sublimates to a patient-centered ecosystem, then we will enjoy stellar outcomes across the board. In addition, by posting the optimal outcomes of surgical resection of non-small cell lung cancer, the true value of surgery compared with increasingly available nonsurgical options is better defined.

Let us choose to lead. Let's decide as a group to find ways to have the tough conversations to float all boats higher sooner, so all patients with lung cancer who come to the surgical arena can benefit from optimal outcomes and reap the benefits that high-quality surgery offers them and their families. We think all patients deserve this opportunity.

\section{References}

1. Lo T, Schiller R, Raghunathan K, Krishnamoorthy V, Jawitz OK, Pyati S, et al. Changes in analgesic strategies for lobectomy from 2009 to 2018. J Thorac Cardiovasc Surg Open. 2021;162:224-36.

2. Cerfolio RJ, Chang SH. Efficiency Quality Index (EQI)_implementing a novel metric that delivers overall institutional excellence and value for patients. Front Surg. 2020;7:604916. 